\title{
AVALIAÇÃO DE FRAGMENTOS DE LENHOS CARBONIZADOS DE ARAUCARIACEAE POR MEIO DE TERMOGRAVIMETRIA E INFRAVERMELHO ASSOCIADAS À ANÁLISE MULTIVARIADA
}

\author{
Daniela M. de Lara ${ }^{\mathrm{a}, \mathrm{b}}$, Laís Bresciani ${ }^{\mathrm{b}}$, Isa Carla Osterkampc, Maurício Hilgemann ${ }^{\mathrm{b}}$, Eduardo Ethurb, André Jasper ${ }^{\mathrm{c} e}$, \\ Marco Flôres Ferrão ${ }^{\mathrm{d}}$, Dieter Uhl ${ }^{\mathrm{e}}$ e Simone Stülp ${ }^{\mathrm{b}, *}$ \\ aUniversidade Estadual do Rio Grande do Sul, Unidade do Alto da Serra Botucaraí Soledade, 99300-000, Soledade - RS, Brasil \\ ${ }^{\mathrm{b} C e n t r o ~ d e ~ C i e ̂ n c i a s ~ E x a t a s ~ e ~ T e c n o l o ́ g i c a s, ~ U n i v a t e s, ~ 95900-000 ~ L a j e a d o ~-~ R S, ~ B r a s i l ~}$ \\ ${ }^{c}$ Centro de Ciências Biológicas e da Saúde, Univates, 95900-000 Lajeado - RS, Brasil \\ 'Instituto de Química, Universidade Federal do Rio Grande do Sul, 91501-970 Porto Alegre - RS, Brasil \\ eSenckenberg Forschungsinstitut und Naturmuseum, 60325, Frankfurt am Main, Germany/Senckenberg Center for Human Evolution \\ and Palaeoenvironment, Institut für Geowissenschaften, Universität Tübingen, 72076, Tübingen, Germany
}

Recebido em 28/12/2016; aceito em 08/05/2017; publicado na web em 19/07/2017

\begin{abstract}
EVALUATION OF CARBONIZED ARAUCARIACEAE WOOD FRAGMENTS WITH THERMOGRAVIMETRY AND FOURIER TRANSFORM INFRARED SPECTROSCOPY ASSOCIATED TO MULTIVARIATE ANALYSIS. The aim of the study is to evaluate the physical and chemical changes that occur on wood fragments submitted to different temperatures, verifying their influence on significant chemical characteristics in the forming process. Carbonization process in muffle associated to the techniques of Thermogravimetry, Fourier Transform Infrared Spectroscopy (FTIR) and multivariate analysis. The analyses were performed on 3 replicates for each burn temperature. The temperature of the thermal muffle process was in every $50{ }^{\circ} \mathrm{C}$, from $200{ }^{\circ} \mathrm{C}$ to $600{ }^{\circ} \mathrm{C}$. TGA were performed under nitrogen atmosphere, using a heating ramp from $25{ }^{\circ} \mathrm{C} \min ^{-1}$ to $995{ }^{\circ} \mathrm{C}$. A range between 1900 to $650 \mathrm{~cm}^{-1}$ was used, with a resolution of $4 \mathrm{~cm}^{-1}$ and 64 scans. The PCA showed that it is possible to describe $95.73 \%$ of the data, grouping the samples into three main clusters. These clusters were used to build a SIMCA (Soft Independent Modeling of Class Analogy) model, enabling to predict with 100\%. Results showed that technical associations, such as TGA, FTIR and multivariate analysis may help to characterize the natural carbonization process and, in future works, contribute to significant (paleo)environmental inferences.
\end{abstract}

Keywords: Araucariaceae; thermogravimetry; mid-infrared; multivariate analysis.

\section{INTRODUÇÃO}

O fogo desempenhou um papel importante em períodos passados da história da Terra, ${ }^{1}$ atuando como um agente onipresente dos distúrbios nos ecossistemas terrestres. ${ }^{2-5}$ Consequentemente, o seu uso proporcionou mudanças nos estilos de vida das comunidades pretéritas, sendo o seu domínio considerado um dos maiores eventos do passado. ${ }^{6}$

As análises dos vestígios deixados pelos incêndios podem auxiliar a inferir condições ambientais predominantes no momento da ocorrência desses eventos, os quais são considerados elementos modeladores de diferentes ecossistemas. ${ }^{7,8}$ Além disso, considerando a relação direta do fogo com o ciclo do carbono, a sua compreensão pode auxiliar no entendimento das dinâmicas ambientais da Terra. ${ }^{3}$

Para que ocorra a combustão de materiais, é imprescindível a ação de três elementos básicos: concentração suficiente de oxigênio atmosférico (comburente), ocorrência de fontes de ignição (energia) e disponibilidade de material combustível. ${ }^{9}$ Quando esses três elementos estão combinados de forma adequada, constitui-se o triângulo do fogo, ${ }^{9}$ sendo que a sua análise integrada possibilita a construção de mosaicos paleoambientais consistentes com base na análise de paleoincêndios. $^{4}$

Os vegetais, após passarem pelo processo de queima, podem não ser completamente consumidos pelo fogo, sendo possível encontrar carvão vegetal ${ }^{5,10}$ como um dos materiais remanescentes. ${ }^{1,5,10-12}$ Assim, a ocorrência de carvão vegetal se constitui em indicativo direto da ocorrência de incêndios atuais ou pretéritos ${ }^{1,5,10-12}$ e o seu estudo reflete

*e-mail: stulp@univates.br as condições ambientais predominantes quando da combustão. ${ }^{8}$

Ainda, técnicas e ferramentas de análise podem igualmente colaborar na elucidação de fenômenos ocorridos na natureza. Através de resultados obtidos por termogravimetria é possível compreender os processos que envolvem reações químicas ou físicas em função da temperatura. Ao encontro disso, o uso de métodos quimiométricos permite construir modelos a partir da relação entre as características físicas e compostos químicos presentes nas análises. Assim, estudos envolvendo técnicas quimiométricas associadas à termogravimetria têm sido relatados com frequência cada vez maior na literatura, e com os mais variados propósitos. Pappa et al. estudaram o efeito de retardadores de fogo sobre os gases formados durante a degradação térmica de celulose. ${ }^{13}$ Gomez et al. estudaram o comportamento da desvolatilização dos lenhos de Pinus e Fagus, provenientes de resíduos de carpintaria e alcachofra (Cynara cardunculus), uma planta energética, assim como o efeito de três pré-tratamentos, lavagem com água quente, extração com etanol e sua combinação. ${ }^{14}$ Risoluti et al. testaram uma nova abordagem para a detecção de $\beta$-thalassemia em sangue de pacientes com desordem genética. ${ }^{15}$ Tomassetti et al. testaram a diferenciação de fósseis humanos muito antigos ( 6.000 A.C.) de fósseis menos antigos (algumas centenas de anos A.C. e D.C.), ${ }^{16} \mathrm{e}$ avaliações por termogravimetria e espectroscopia associada à análise multivariada são ferramentas que vêm sendo adotadas para avaliação das características físico-químicas de carvões vegetais ${ }^{17-25} \mathrm{e}, \mathrm{em}$ consequência, dos (paleo)incêndios vegetacionais.

Estudos desenvolvidos utilizando tais ferramentas apresentaram características químicas significativas, contribuindo para uma fonte valiosa de dados proxy (paleo)ambientais. ${ }^{21,26-28}$ Por outro lado, estudos que se concentram na caracterização físico-química de 
carvões vegetais são raros, principalmente aqueles relacionados a Araucariaceae.

Neste sentido, o objetivo do presente trabalho é avaliar as alterações físico-químicas que ocorrem durante o processo de carbonização em mufla de fragmentos de lenhos (Araucariaceae), em diferentes temperaturas, permitindo avaliar a sua influência sobre características químicas dos carvões vegetais. Para tanto, os lenhos foram avaliados por termogravimetria e infravermelho médio associadas à análise multivariada. A análise destes resultados busca permitir a definição de uma metodologia que auxilie na caracterização dos processos naturais envolvidos na formação desse tipo de material, servindo de subsídio para inferências (paleo)ambientais.

\section{PARTE EXPERIMENTAL}

\section{Identificação das amostras}

Fragmentos de lenhos de três espécies de Araucariaceae foram coletados em diferentes localidades do Rio Grande do Sul, Brasil. Foram extraídas de espécimes abatidas por motivos diversos em áreas dos municípios de São José dos Ausentes (28 47’ 06,56" S e $49^{\circ} 58^{\prime}$ 50,85" L - Araucaria angustifolia), Novo Cabrais ( $29^{\circ} 47^{\prime} 3,48^{\prime \prime}$ S e $52^{\circ} 58^{\prime} 14,59^{\prime \prime}$ L - Araucaria bidwillii) e Colinas (293' $28,84^{\circ}$ ' S e $51^{\circ} 50^{\prime} 28,35^{\prime}$ ' L - Araucaria columnaris).

As amostras foram obtidas em forma de discos a 1,50 m de altura, medindo $8 \mathrm{~cm}$ de espessura e 18 a $35 \mathrm{~cm}$ de diâmetro.

Os lenhos secos foram identificados de duas formas diferentes, antes e depois do processo de carbonização. As amostras que antecedem a queima foram identificadas como lenhos in natura e, após o processo de queima, as amostras foram identificadas como lenhos carbonizados artificialmente.

\section{Caracterização dos lenhos in natura por termogravimetria}

As medidas termogravimétricas para as três espécies foram realizadas utilizando uma termobalança Perkin Elmer, modelo TGA-4000, sob atmosfera de nitrogênio gasoso, vazão constante de $20 \mathrm{~mL} \mathrm{~min}^{-1}$, em amostras de $20 \mathrm{mg}( \pm 2 \mathrm{mg})$ dos lenhos in natura. As análises foram realizadas em 6 replicatas para cada espécie avaliada. A razão de aquecimento foi de $25^{\circ} \mathrm{C} \mathrm{min}{ }^{-1}$ até obter a temperatura final de $995^{\circ} \mathrm{C}$.

O percentual de massa residual, durante o processo carbonização, foi calculado a partir da massa inicial de cada amostra subtraindo-se o valor da massa final.

\section{Processo de carbonização em mufla}

Os lenhos in natura foram carbonizados em mufla SP Labor (modelo SP-1200). As amostras foram cortadas com dimensões de $1,0 \mathrm{~cm} \times 1,0 \mathrm{~cm} \times 1,0 \mathrm{~cm}\left(1,0 \mathrm{~cm}^{3}\right)$, com variância de $0,02 \mathrm{~cm}$. Posteriormente, no cadinho de porcelana (Chiarotti A-37) foi depositada uma quantidade de areia suficiente para preencher o fundo do mesmo. Após essa etapa, a amostra previamente pesada foi colocada dentro do cadinho de porcelana e, objetivando restringir a quantidade de oxigênio no processo de carbonização, o recipiente foi preenchido até sua borda com areia do mar purificada (Merck), cujo tamanho de partículas variam de 0,1 a $0,3 \mathrm{~mm}$.

As temperaturas da queima dos lenhos in natura foram feitas num intervalo de 200 a $600{ }^{\circ} \mathrm{C}$, variando-se a temperatura de 50 em $50{ }^{\circ} \mathrm{C}$ e com razão de aquecimento avaliada em $25^{\circ} \mathrm{C} \mathrm{min}^{-1}$. As análises foram realizadas em triplicata para cada temperatura de queima $\mathrm{e}$ para cada uma das três espécies avaliadas, totalizando 81 amostras.

Para o processo de carbonização, a temperatura de aquecimento do forno mufla era regulada a $50{ }^{\circ} \mathrm{C}$ por 5 minutos para estabilização.
Após esse tempo, as amostras foram colocadas no interior da mufla, ajustando-se a temperatura desejada. Quando o equipamento atingia a temperatura estipulada, a amostra permanecia no forno por 60 minutos. Posterior a esse período, as amostras eram retiradas da mufla e acondicionadas em dessecador para que atingissem a temperatura ambiente.

As perdas de massas durante o processo de carbonização foram calculadas a partir da massa inicial de cada amostra subtraindo-se o valor da massa final obtida e expressas em porcentagem.

\section{Análises estatísticas}

Os dados de massa residual percentual para as termogravimetrias e os dados de perda de massa para o processo de carbonização em mufla foram submetidos à análise de variância de um fator (one-way ANOVA) utilizando o software Bioestat 5.3 e, quando estabelecidas diferenças significativas, os tratamentos foram comparados entre si por meio do teste de Tukey para os níveis de significância de $1 \%$ $(\mathrm{p}<0,01)$ e $5 \%(\mathrm{p}<0,05)$.

\section{Obtenção dos espectros de IR}

Os espectros foram adquiridos no espectrofotômetro SHIMADZU, modelo IR Affinity-1. Antecedendo as leituras dos espectros, foi realizado a preparação das amostras de lenhos carbonizados artificialmente a partir da confecção da pastilha de KBr. Subsequente à fabricação da pastilha, os espectros foram adquiridos no espectrofotômetro de infravermelho, num intervalo entre 1900 a $650 \mathrm{~cm}^{-1}$, com resolução de $4 \mathrm{~cm}^{-1}$ e 64 varreduras.

Para a aquisição dos espectros no infravermelho médio foi realizada primeiramente a leitura do branco (background). Os espectros para cada amostra foram adquiridos em triplicata.

\section{Análise multivariada}

A análise de componentes principais (PCA) foi realizada com o software Chemostat..$^{29}$ Os dados dos espectros de FTIR obtidos foram previamente suavizados pelo método de Savitsky-Golay, centrados na média e aplicada a primeira derivada antes de serem submetidos à PCA. A PCA foi realizada utilizando-se um total de 54 amostras (2 replicatas por temperatura avaliada, por espécie analisada), que serviram como conjunto de calibração. O modelo foi validado pelo método de validação cruzada.

A modelagem SIMCA (modelagem independente e flexível por analogia de classe) foi realizada com o software The Unscrambler X (CAMO AS), com base nos modelos de PCA criados para cada classe de amostras observadas no conjunto de calibração. Os modelos foram validados pelo método de validação cruzada, e aplicou-se a modelagem SIMCA para prever as temperaturas de carbonização de 27 amostras (1 amostra por temperatura avaliada, por espécie analisada) que serviram como conjunto de predição.

\section{RESULTADOS E DISCUSSÃO}

\section{Caracterização dos lenhos in natura por termogravimetria}

A Figura 1 apresenta as medidas termogravimétricas médias para as três espécies avaliadas.

Observa-se que, para as três espécies, a termodegradação dos lenhos ocorre em eventos térmicos definidos e padrões de queima semelhantes (regiões 1, 2, 3 e 4 da Figura 1a). Ainda, em destaque na mesma figura, evidenciam-se as variações de perda de massa entre as medidas termogravimétricas para as espécies avaliadas.

Verifica-se que ocorre perda de massa no intervalo de $25^{\circ} \mathrm{C}$, temperatura ambiente, até $150^{\circ} \mathrm{C}$. A média da perda de massa das três 

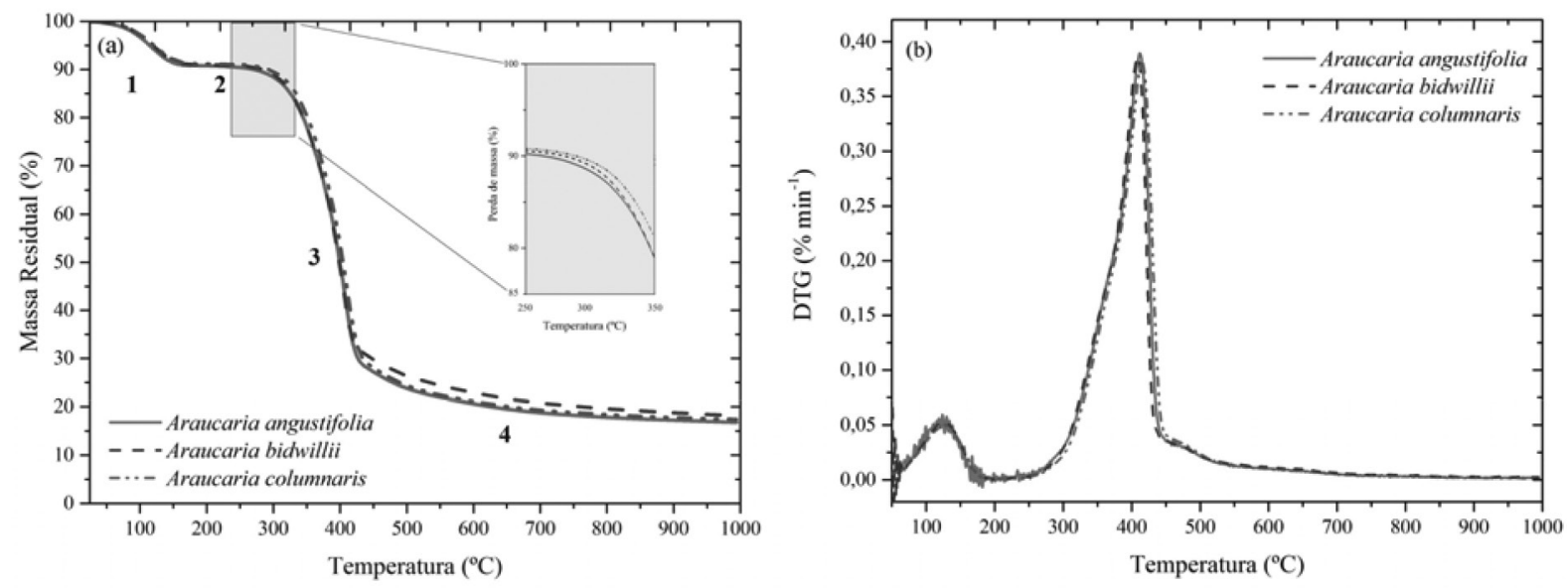

Figura 1. Medidas termogravimétricas das espécies Araucaria angustifolia, Araucaria bidwillii e Araucaria columnaris com razão de aquecimento de $25^{\circ} \mathrm{C}$ min ${ }^{-1}$ e faixa de temperatura de aquecimento de $25^{\circ} \mathrm{C}$ a $995{ }^{\circ} \mathrm{C}$ (curvas TG) com regiões 1,2 (detalhe em zoom), 3 e 4 destacados (a) e curvas DTG (b)

espécies na faixa de temperatura de $25^{\circ} \mathrm{C}$ até $150{ }^{\circ} \mathrm{C}$ foi de $8,58 \%$, $8,17 \%$, e $7,93 \%$ para as espécies A. angustifolia, A. bidwillii e A. columnaris, respectivamente, não havendo diferenças significativas entre elas.

O intervalo de temperatura de $200{ }^{\circ} \mathrm{C}$ até $300{ }^{\circ} \mathrm{C}$ (região 2 Figura 1a) apresenta uma região de estabilidade térmica no perfil termogravimétrico das três espécies.

A maior perda de massa se deu no intervalo de $300^{\circ} \mathrm{C}$ até $450{ }^{\circ} \mathrm{C}$ (região 3 da Figura 1a e Figura 1b). As médias das perdas de massa foram $61,22 \%, 59,26 \%$, e 61,52\% para as espécies A. angustifolia, A. bidwillii e A. columnaris, respectivamente. Observou-se que entre as espécies $A$. angustifolia e A. bidwillii e A. bidwillii e A. columnaris houve diferenças significativas com $\mathrm{p}<0,05$ e $\mathrm{p}<0,01$, respectivamente.

No intervalo de $450^{\circ} \mathrm{C}$ até $995^{\circ} \mathrm{C}$ (região 4 da Figura 1a) as perdas de massa média foram $10,42 \%$ para $A$. angustifolia, 11,64\% para $A$. bidwillii e $10,59 \%$, para $A$. columnaris. Novamente, observou-se que apenas entre as espécies $A$. angustifolia e A. bidwillii e A. bidwillii e $A$. columnaris houve diferenças significativas com $\mathrm{p}<0,01$ para ambos os casos.

Após a obtenção da temperatura máxima de $995{ }^{\circ} \mathrm{C}$, a massa residual, em termos percentuais, para as espécies A. angustifolia, A. bidwillii e A. columnaris foram de $16,80 \%, 18,15 \%$ e $17,45 \%$, respectivamente, havendo diferença significativa apenas entre as espécies A. angustifolia e A. bidwillii ( $\mathrm{p}<0,05)$.

As varreduras por termogravimetria dos lenhos secos in natura indicam que, quando submetidos à ação do calor, sofrem processo de transformação, nos quais seus componentes são modificados. Características como redução de massa, fragmentação e arredondamento dos materiais carbonizados são observados. ${ }^{30,31}$

Com o aumento da temperatura, modificações químicas ocorrem nos lenhos. Na fase inicial de aquecimento, entre $20^{\circ} \mathrm{C}$ e $110^{\circ} \mathrm{C}$, o lenho absorve calor e libera vapor d'água. A $270^{\circ} \mathrm{C}$ o lenho começa a degradar e ainda existem resquícios remanescentes de água que continuam sendo liberados juntamente com compostos, tais como: monóxido de carbono, dióxido de carbono, ácido acético e metanol. ${ }^{32}$

$\mathrm{Na}$ fase de aquecimento do lenho (faixa de $270{ }^{\circ} \mathrm{C}$ a $290{ }^{\circ} \mathrm{C}$ ), inicia-se o processo exotérmico, e de $290{ }^{\circ} \mathrm{C}$ a $400{ }^{\circ} \mathrm{C}$, o lenho continua se degradando, liberando gases combustíveis e vapores condensáveis. ${ }^{28} \mathrm{~A} 400{ }^{\circ} \mathrm{C}$ a transformação dos lenhos é praticamente completa. ${ }^{32}$

No intervalo de $300{ }^{\circ} \mathrm{C}$ a $450{ }^{\circ} \mathrm{C}$ (Figura 1b) acontece a maior perda de massa durante o processo de termodegradação dos lenhos. Para o entendimento das alterações físico-químicas ocorridas nos lenhos após o processo de carbonização devem ser considerados os seus três principais constituintes: celulose, hemicelulose e lignina. ${ }^{33}$ A combinação de celulose (40-45\%) e as hemiceluloses (15-25\%), conhecida como holocelulose, geralmente representa $65-70 \%$ da massa seca dos lenhos. ${ }^{34}$

As hemiceluloses são termicamente menos estáveis se comparadas aos demais componentes dos lenhos, devido à sua estrutura ${ }^{35} \mathrm{e} a$ sua decomposição térmica pode variar para taxas máximas de perda de massa entre $243{ }^{\circ} \mathrm{C}$ e $332{ }^{\circ} \mathrm{C} .{ }^{36}$

Estudos desenvolvidos utilizando termogravimetria em lenhos de Castanea sativa (angiosperma) e Pinus pinaster (gimnosperma) permitiram identificar características da holocelulose de ambas as espécies. ${ }^{25}$ As medidas termogravimétricas observadas apresentaram um comportamento térmico semelhante, com pico a $350{ }^{\circ} \mathrm{C}$ e um shoulder a $280{ }^{\circ} \mathrm{C} .{ }^{25}$ Os autores concluíram que, como a holocelulose é composta de celulose e hemicelulose, o pico a $350{ }^{\circ} \mathrm{C}$ poderia, então, ser facilmente atribuído à celulose, e o shoulder a $280^{\circ} \mathrm{C}$ para a holocelulose. ${ }^{25} \mathrm{~A}$ principal diferença entre as duas espécies foi o pico ligeiramente mais estreito na espécie Pinus pinaster e, assim, o shoulder é mais evidente neste caso. ${ }^{25} \mathrm{O}$ estudo indica, ainda, a presença de componentes diferentes nas duas espécies, os quais afetariam a estabilidade térmica dos seus constituintes. ${ }^{25}$

Análises em lenhos de eucalipto por processos de pirólise e por combustão objetivaram simular de forma aproximada os principais constituintes dos seus lenhos (hemicelulose, celuloses cristalinas e amorfas, lignina, dentre outros) ${ }^{37} \mathrm{O}$ processo de degradação por pirólise demonstrou que a perda de massa ocorre em somente uma etapa, sendo basicamente a consequência das volatilizações de hemicelulose (shoulder $299^{\circ} \mathrm{C}$ ) e celulose (pico a $363^{\circ} \mathrm{C}$ ). Já em processos termodegradativos (em ambientes com presença de ar), a carbonização dos lenhos de eucalipto apresentou duas etapas de perda de massa. A primeira etapa é semelhante ao observado em condições de pirólise, mas ocorre a temperaturas mais baixas, mostrando uma maior taxa de perda de massa. Neste caso, as degradações de hemicelulose e celulose ocorrem a $294^{\circ} \mathrm{C}$ e $328^{\circ} \mathrm{C}$, respectivamente. $\mathrm{O}$ segundo pico, causado pela oxidação do carvão, ocorre próximo a $450{ }^{\circ} \mathrm{C} .{ }^{37}$

Estudos comparativos de análise do processo de carbonização de madeira e celulose de pinus, além de Lignina Brauns, também foram realizados. ${ }^{38}$ Foi demonstrado que a madeira teve a decomposição de celulose (pico de $300{ }^{\circ} \mathrm{C}$ ) e de lignina (pico de $420^{\circ} \mathrm{C}$ ). Além disso, enquanto a celulose purificada apresentou pequena quantidade de lignina (pico de $420{ }^{\circ} \mathrm{C}$ ) remanescente, a Lignina Brauns possuía algum percentual $\left(7 \%\right.$ ) de hemicelulose (pico de $240{ }^{\circ} \mathrm{C}$ )..$^{38}$

Esses resultados foram utilizados como base para a análise de alterações químicas observadas durante o processo de carbonização de 
madeira na formação de carvão vegetal ${ }^{38}$ Interpretou-se que, durante o processo de queima, a madeira sofre alterações em sua constituição, sendo que a celulose é facilmente degradada nas temperaturas entre $200{ }^{\circ} \mathrm{C}$ e $340{ }^{\circ} \mathrm{C} .{ }^{38}$ Por outro lado, apesar de a degradação da lignina iniciar a temperaturas relativamente baixas $\left(150^{\circ} \mathrm{C}\right)$, ela prossegue lentamente por um amplo gradiente de temperaturas, podendo se prolongar até os $900{ }^{\circ} \mathrm{C} . .^{39-41}$

A estabilidade da lignina e a baixa taxa de perda de massa a ela relacionada foi vinculada à sua aromaticidade e ao tamanho e arranjo na estrutura molecular. ${ }^{38-41}$ Estas características se opõem às da celulose e da hemicelulose, que possuem um arranjo estrutural mais simples e temperatura de degradação abaixo de $350{ }^{\circ} \mathrm{C} .{ }^{38,39}$

\section{Carbonização dos lenhos in natura em mufla}

A Figura 2 apresenta os resultados em termos de perda de massa (\%) das amostras avaliadas em mufla para a faixa de temperatura de $200{ }^{\circ} \mathrm{C}$ a $600{ }^{\circ} \mathrm{C}$ para as três espécies avaliadas.

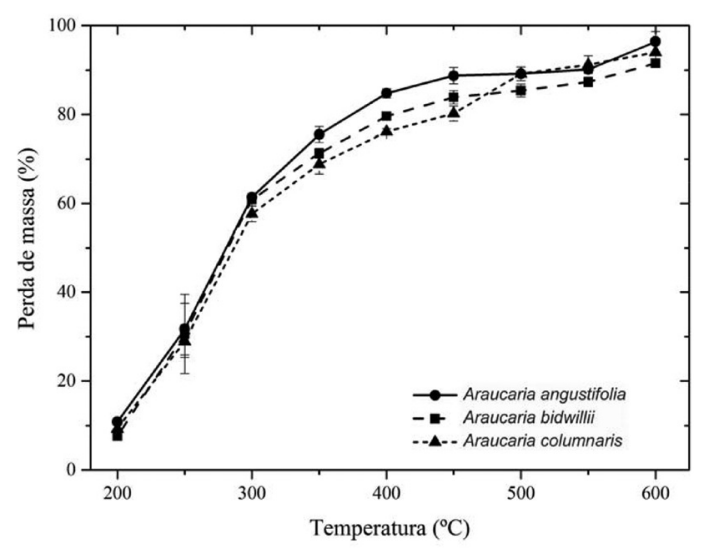

Figura 2. Perda de massa das amostras submetidas ao processo de queima em mufla para as espécies Araucaria angustifolia, Araucaria bidwillii e Araucaria columnaris na faixa de temperatura de $200{ }^{\circ} \mathrm{C}$ a $600{ }^{\circ} \mathrm{C}$

Observa-se, na Figura 2, eventos térmicos similares e que apresentaram médias de perda de massa durante o processo de termodegradação a $600{ }^{\circ} \mathrm{C}$ de $96,34 \%, 91,60 \%$ e $94,03 \%$ para as espécies A. angustifolia, A. bidwillii e A. columnaris, respectivamente, sendo que apenas a diferença entre as espécies $A$. angustifolia e $A$. bidwillii mostrou-se estatisticamente significativa $(\mathrm{p}<0,05)$.

No intervalo inicial, de $200^{\circ} \mathrm{C}$ a $300^{\circ} \mathrm{C}$, os lenhos apresentaram a maior perda de massa. Os resultados médios para as perdas de massa foram $50,57 \%$ para $A$. angustifolia, $53,21 \%$ para $A$. bidwillii e $48,35 \%$ para $A$. columnaris.

No intervalo de $300{ }^{\circ} \mathrm{C}$ até $450{ }^{\circ} \mathrm{C}$ ocorre uma diminuição na perda de massa para as três espécies se comparado aos dois estágios anteriores, apresentando perdas de massa de 27,39\%, 23,06\% e $22,59 \%$, respectivamente, para as espécies A. angustifolia, A. bidwillii e A. columnaris.

De forma análoga aos resultados apresentados na Figura 1, os lenhos secos in natura analisados não receberam tratamento térmico que antecedesse as análises físicas e químicas. Através da proposição da metodologia de carbonização dos lenhos in natura em mufla para as espécies A. angustifolia, A. bidwillii e A. columnaris, observou-se que a $A$. angustifolia foi a que mais perdeu massa, sucedida da $A$. columnaris e $A$. bidwillii, sendo que a maior perda de massa se deu entre $250{ }^{\circ} \mathrm{C}$ e $300{ }^{\circ} \mathrm{C}$.

Concomitantemente nessa faixa de temperatura houve a mudança de coloração dos lenhos carbonizados artificialmente de marrom escuro para preto. Ao reduzir o teor de umidade das amostras, inicia-se o processo de decomposição da estrutura molecular dos lenhos. A umidade da biomassa provém da água, que faz parte da composição da estrutura macro do lenho e está ligada às paredes de celulose. A perda dessa água causa tensão na estrutura molecular e se manifesta macroscopicamente pela mudança da sua coloração para preto. ${ }^{42}$

As variabilidades das características (tamanho e a forma do lenho, umidade e a razão de aquecimento) que ocorrem no processo de carbonização do carvão são fatores que influenciam no processo de formação do mesmo. ${ }^{43}$ Além de que o lenho, por ser um polímero natural, quando submetido ao fogo, sofre despolimerização e carbonização, alterando suas propriedades mecânicas conforme o nível de temperatura e tempo de exposição ao calor ao qual foi submetida. ${ }^{44}$ Ainda, alguns autores citam que as variações de massa entre as espécies e dentre a mesma espécie podem ocorrer em função da diferença de idade entre os indivíduos, ${ }^{45,46}$ fato este observado no detalhe apresentado na Figura 1.

Ressalta-se que os dois processos térmicos, termodegradação em mufla e em termogravimetria, são distintos e não comparativos, uma vez que em mufla as amostras foram carbonizadas em uma atmosfera autogerada com próprios gases liberados na carbonização, condição contrária à das amostras carbonizadas por termogravimetria, em que as amostras são cuidadosamente pesadas enquanto sofrem o processo de termodegradação em um ambiente isento de oxigênio, com controle de gás inerte e razão de aquecimento programada a fim de obter medidas térmicas para as três espécies avaliadas.

\section{Representação dos grupos funcionais por FTIR}

Representando um conjunto de dados de 81 espectros, 3 espectros são exibidos nas Figuras 3a e 3b na região espectral de 1900 e $650 \mathrm{~cm}^{-1}$. Considerou-se para a escolha dos mesmos as temperaturas que exibiram alterações mais significativas nos espectros por faixa de temperatura e por espécie avaliada.

A Figura 3a exibe três temperaturas distintas, 200, 300 e $600{ }^{\circ} \mathrm{C}$, representando A. columnaris, A. angustifolia e A. bidwillii, respectivamente. Ao avaliar individualmente os conjuntos dos espectros por espécie, após a normalização da linha base (Figura 3b), observa-se que as três espécies da Araucariaceae exibem uma semelhança espectral característica por faixa de temperatura. As bandas com maior intensidade estão localizadas a partir de $1800 \mathrm{~cm}^{-1}$ se estendendo até $1500 \mathrm{~cm}^{-1}$, para temperaturas de carbonização acima de $300^{\circ} \mathrm{C}$, faixa espectral atribuída aos grupos funcionais $v \mathrm{C}=\mathrm{O}$ e $v \mathrm{C}=\mathrm{C} .{ }^{47}$

A representação dos lenhos carbonizados em $200{ }^{\circ} \mathrm{C}$ pode ser observada nos espectros da A. columnaris na Figura 3a. Os picos representativos nesta faixa de temperatura são: $1738 \mathrm{~cm}^{-1} \mathrm{e} 1651 \mathrm{~cm}^{-1}$ pertencentes ao grupo funcional $v \mathrm{C}=\mathrm{O}, 1508 \mathrm{~cm}^{-1}$ atribuído à absorção de compostos aromáticos $(v \mathrm{C}=\mathrm{C}), 1465 \mathrm{~cm}^{-1}$ relacionado a absorção $\delta \mathrm{CH}_{2}, 1424 \mathrm{~cm}^{-1}$ e $1372 \mathrm{~cm}^{-1}$ atribuídos ao grupo vibracional $\delta \mathrm{CH}_{3}$, $1267 \mathrm{~cm}^{-1}, 1157 \mathrm{~cm}^{-1}, 1111 \mathrm{~cm}^{-1}, 1059 \mathrm{~cm}^{-1}$ e $1030 \mathrm{~cm}^{-1}$ elencados ao grupo funcional $v \mathrm{C}-\mathrm{O}$ e, $897 \mathrm{~cm}^{-1}, 810 \mathrm{~cm}^{-1}$ e $673 \mathrm{~cm}^{-1}$ atribuídos ao grupo $\gamma \mathrm{C}-\mathrm{H} .{ }^{47}$

Os picos representativos em $300^{\circ} \mathrm{C}$ para os lenhos carbonizados da espécie A. angustifolia são: $1717 \mathrm{~cm}^{-1} \mathrm{e} 1705 \mathrm{~cm}^{-1}$ pertencentes ao grupo funcional $\nu \mathrm{C}=\mathrm{O}, 1601 \mathrm{~cm}^{-1} \mathrm{e} 1508 \mathrm{~cm}^{-1}$ atribuídos às absorções dos compostos aromáticos $(v \mathrm{C}=\mathrm{C}), 1424 \mathrm{~cm}^{-1}$ e $1366 \mathrm{~cm}^{-1}$ atribuídos ao grupo vibracional $\delta \mathrm{CH}_{3}, 1229 \mathrm{~cm}^{-1}$ elencado ao grupo funcional $v \mathrm{C}-\mathrm{O}$ e, $837 \mathrm{~cm}^{-1}, 772 \mathrm{~cm}^{-1}$ e $673 \mathrm{~cm}^{-1}$ atribuídos ao grupo $\gamma \mathrm{C}-\mathrm{H} \cdot{ }^{47}$

As características espectrais das amostras $200{ }^{\circ} \mathrm{C}$ e $300^{\circ} \mathrm{C}$ distinguem-se das amostras carbonizadas artificialmente em $600{ }^{\circ} \mathrm{C}$. Os picos representativos dos lenhos carbonizados artificialmente da A. bidwillii são: $1700 \mathrm{~cm}^{-1}$ pertencente ao grupo funcional $v \mathrm{C}=\mathrm{O}$, $1560 \mathrm{~cm}^{-1}$ e $1541 \mathrm{~cm}^{-1}$ atribuídos ao grupo funcional $\nu \mathrm{C}=\mathrm{C}, 1424 \mathrm{~cm}^{-1}$ 

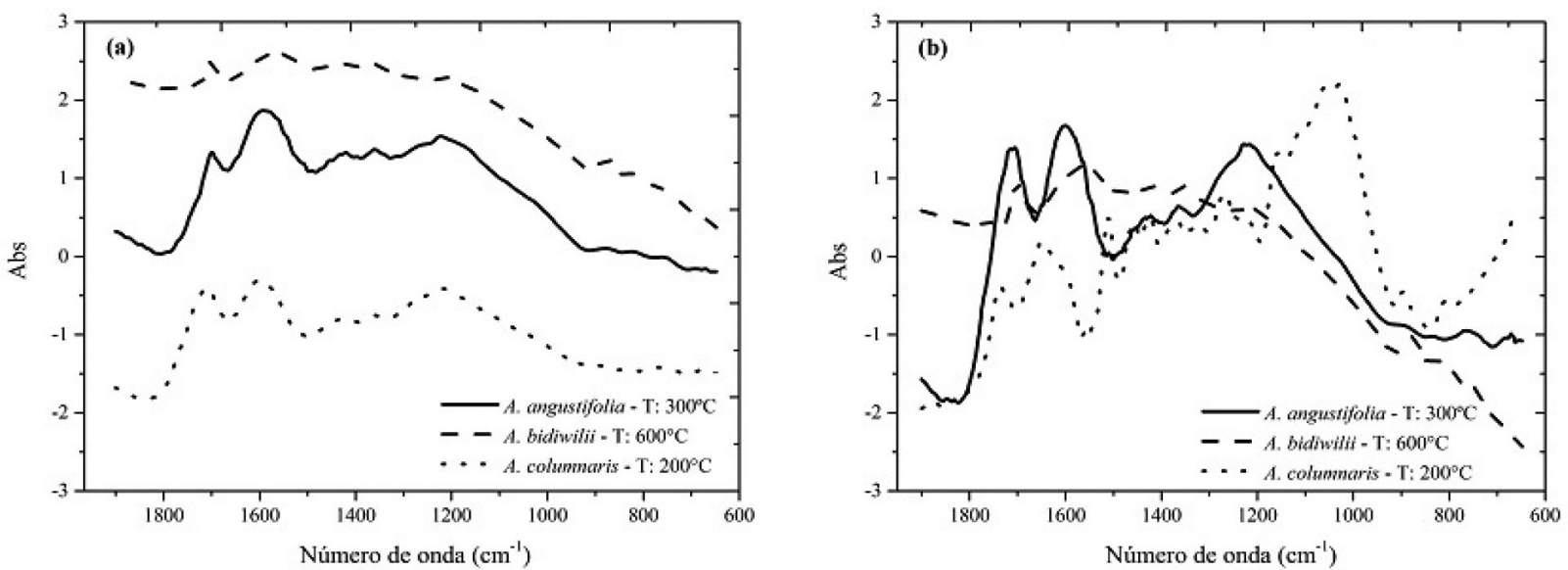

Figura 3. Representação do conjunto espectral dos lenhos carbonizados artificialmente em mufla para as três espécies avaliadas na faixa espectral entre 650 a $1900 \mathrm{~cm}^{-1}$ (a) dados originais (b) após normalização da linha base

relacionado ao grupo funcional $\delta \mathrm{CH}_{3}, 1350 \mathrm{~cm}^{-1}$ elencado ao grupo $\mathrm{S}=\mathrm{O}, 1220 \mathrm{~cm}^{-1}$ elencado ao grupo funcional $v \mathrm{C}-\mathrm{O}$ e, $876 \mathrm{~cm}^{-1}$, $822 \mathrm{~cm}^{-1}, 810 \mathrm{~cm}^{-1}$ e $752 \mathrm{~cm}^{-1}$ atribuídos ao grupo funcional $\gamma \mathrm{C}-\mathrm{H} \cdot{ }^{47}$ $\mathrm{O}$ detalhamento dos principais picos presentes nos espectros na Figura $3 \mathrm{~b}$ está apresentado na Tabela $1 \mathrm{~S}$.

Ainda, a fim de obter maiores informações, a análise multivariada foi utilizada considerando a quantidade de informações espectrais entre 1900 a $650 \mathrm{~cm}^{-1}$.

\section{Caracterização por análise de componentes principais}

A PCA foi usada no conjunto de calibração para focar a similaridade dos dados obtidos a partir das análises por FTIR. Considerando esta abordagem, os melhores resultados para a PCA foram obtidos após os espectros serem suavizados pelo método de Savitsky-Golay, normalizados, serem centrados na média e ser aplicada a primeira derivada. A PCA mostrou que com seis componentes principais é possível descrever $95,73 \%$ dos dados. Assim, o gráfico dos scores (Figura 4) contém a maior parte da variância explicada $(78,18 \%)$ agrupando as amostras em três grupos principais, uma vez que dois pontos localizados próximos entre si devem conter informações espectrais semelhantes.

A análise do gráfico dos scores (Figura 4) mostra as amostras separadas em diferentes grupamentos. A PC1 separa as amostras com temperaturas de carbonização abaixo de $250{ }^{\circ} \mathrm{C}$ (grupo 1) em valores positivos ao longo do vetor, das amostras cuja carbonização foi realizada acima de $300^{\circ} \mathrm{C}$, que possuem valores negativos ao longo desta PC. Essa separação se dá principalmente devido às variações na altura dos sinais de $\log (1 / \mathrm{R})$ situados próximos a $1713 \mathrm{~cm}^{-1}$, $1522 \mathrm{~cm}^{-1}, 1171 \mathrm{~cm}^{-1}$ e $982 \mathrm{~cm}^{-1}$. Isto se torna mais evidente ao se analisar o gráfico dos loadings referentes à PC1 (Figura 5). Os maiores valores absolutos de $\log (1 / \mathrm{R})$ para os loadings da PC1 localizam-se nas regiões espectrais próximas a $1710 \mathrm{~cm}^{-1}, 1520 \mathrm{~cm}^{-1}, 1170 \mathrm{~cm}^{-1} \mathrm{e}$ $980 \mathrm{~cm}^{-1}$. O distanciamento entre as amostras carbonizadas a $250{ }^{\circ} \mathrm{C}$ pode estar atrelado ao maior desvio observado para os valores de perda de massa apresentados na Figura 2.

Já a PC2 (Figura 4) diferencia as amostras que apresentam valores negativos no vetor $\mathrm{PC} 1 \mathrm{em}$ dois subgrupos, um consistindo em amostras com temperaturas de carbonização entre $300{ }^{\circ} \mathrm{C}$ e $400{ }^{\circ} \mathrm{C}$ (grupo 2), e outro em que as amostras foram carbonizadas em temperaturas acima de $500{ }^{\circ} \mathrm{C}$ (grupo 3), em valores negativos e positivos ao longo do vetor, respectivamente. Observando-se o gráfico dos loadings desta PC (Figura 5), percebe-se que essa distinção se faz em função das variações na altura dos sinais de $\log (1 / \mathrm{R})$ situados nas regiões de $1730-1790 \mathrm{~cm}^{-1}$ e $1540-1570 \mathrm{~cm}^{-1}$.

A maioria das amostras carbonizadas a $450{ }^{\circ} \mathrm{C}$ apresentaram

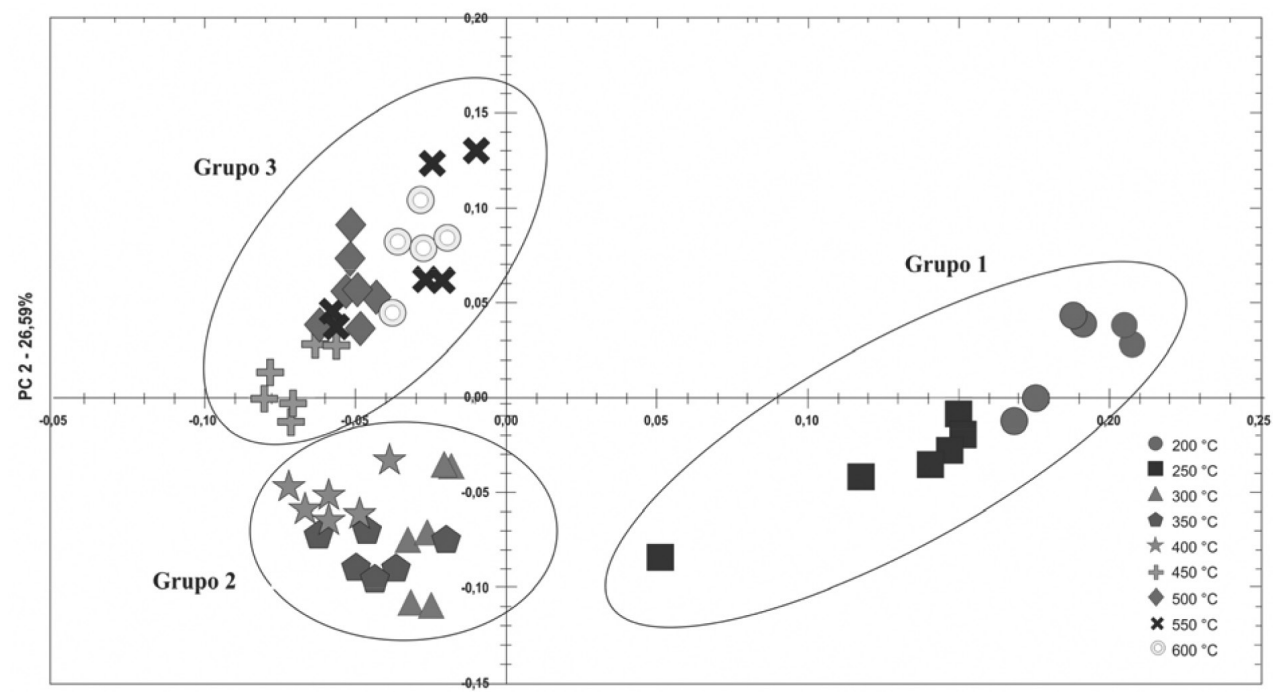

PC $1 \cdot 51,59 \%$

Figura 4. Representação dos scores para PC1 versus PC2 para as amostras de lenhos carbonizados artificialmente em mufla para a A. angustifolia, A. bidwillii e A. columnaris 

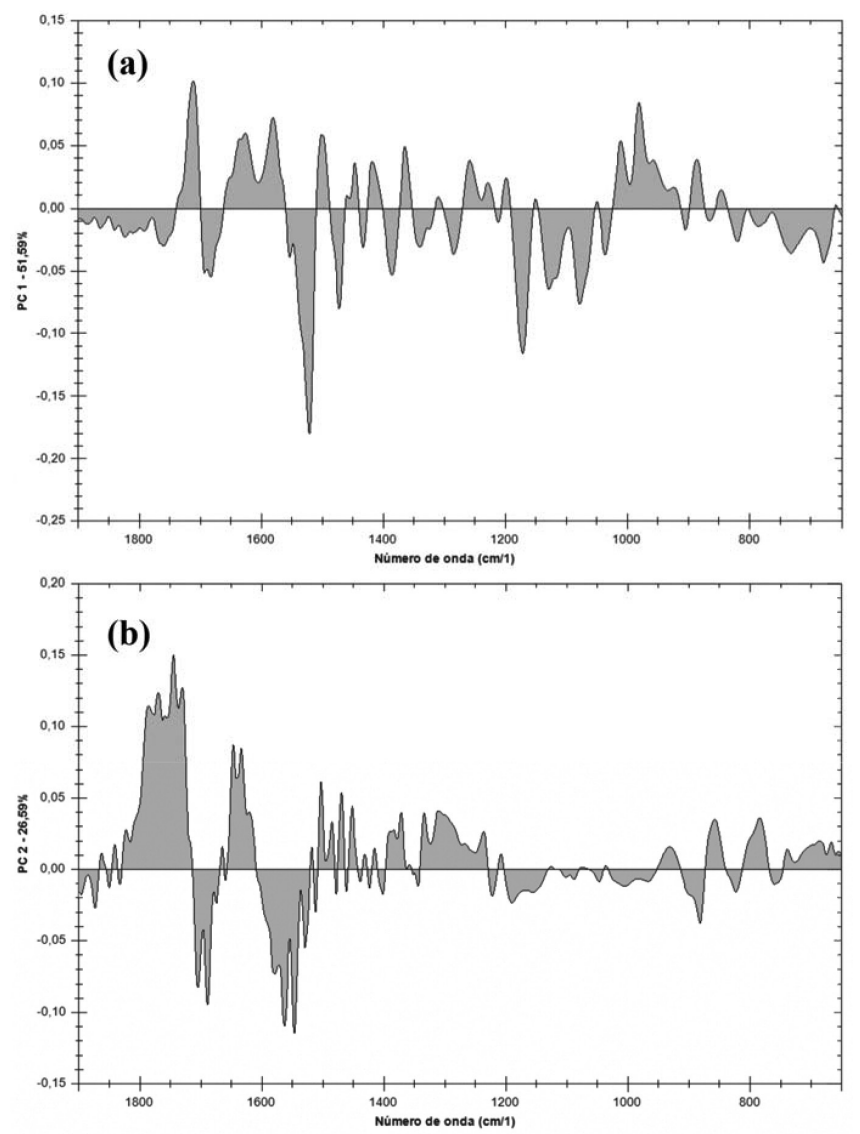

Figura 5. Loadings da componente principal 1 (a) e da componente principal 2 (b) para as amostras de lenhos carbonizados artificialmente em mufla para as três espécies de Araucariaceae avaliadas

valores pequenos para o vetor PC2, de modo que se encontram próximas à origem. No entanto, elas foram adicionadas ao grupo 3, visto que a maioria destas amostras apresenta valores positivos para a PC2. Assim, verifica-se que com o aumento da temperatura há a formação de distintos agrupamentos: temperaturas de carbonização abaixo de $250{ }^{\circ} \mathrm{C}$ (grupo 1), temperaturas de carbonização entre $300^{\circ} \mathrm{Ce} 400^{\circ} \mathrm{C}$ (grupo 2) e temperaturas de carbonização acima de $450^{\circ} \mathrm{C}$ (grupo 3). Além disso, observa-se que o modelo multivariado não faz distinção entre as três espécies avaliadas (Figura 1S).

Ao avaliar os resultados espectrais obtidos na análise por FTIR e, consequentemente, a formação dos grupos na Figura 4 por análise multivariada, observa-se que os lenhos carbonizados artificialmente para as três espécies apresentaram um comportamento similar ao $2^{\circ}$, $3^{\circ} \mathrm{e} 4^{\circ}$ eventos térmicos apresentados nas medidas termogravimétricas (Figura 1), os quais retratam mudanças físicas significativas concomitantemente com as mudanças químicas dos lenhos.

\section{SIMCA}

Análises de componentes principais independentes foram realizadas para cada grupo no conjunto de calibração para construir os modelos de classe para os grupos $1(n=12), 2(n=18)$ e $3(n=24)$. Para todas as classes, 6 componentes principais foram escolhidas para uma variância total explicada de $98,7 \%, 96,2 \%$ e $97,1 \%$ para os modelos 1,2 e 3 , respectivamente.

As distâncias de 192,6 entre os modelos 1 e 2, de 367,1 entre os modelos 1 e 3 e de 43,6 entre os modelos 2 e 3 indicam uma separação muito boa, sendo 3 o limite para considerar duas classes significativamente separadas para fins de identificação.
O conjunto de predição foi utilizado para a validação externa. Este conjunto contém 27 amostras, contendo elementos de todas as espécies analisadas, em todas as temperaturas avaliadas. Como pode ser observado na Tabela 1 , todos os modelos apresentaram $100 \%$ de classificação correta das respectivas classes modeladas, com nível de significância de $10 \%$ para as 27 amostras do grupo de predição.

Tabela 1. Resultados da modelagem SIMCA para o conjunto de predição a um nível de significância de $10 \%$

\begin{tabular}{|c|c|c|c|}
\hline Amostras & $\begin{array}{c}\text { Grupo } 1 \\
\left(200-250^{\circ} \mathrm{C}\right) \\
\end{array}$ & $\begin{array}{c}\text { Grupo } 2 \\
\left(300-400{ }^{\circ} \mathrm{C}\right) \\
\end{array}$ & $\begin{array}{c}\text { Grupo } 3 \\
\left(450-600{ }^{\circ} \mathrm{C}\right)\end{array}$ \\
\hline A. biwilli_200 & $*$ & & \\
\hline A. biwilli_250 & $*$ & & \\
\hline A. biwilli_300 & & $*$ & \\
\hline A. biwilli_350 & & $*$ & \\
\hline A. biwilli_400 & & $*$ & \\
\hline A. biwilli_450 & & & $*$ \\
\hline A. biwilli_500 & & & $*$ \\
\hline A. biwilli_550 & & & $*$ \\
\hline A. biwilli_600 & & & $*$ \\
\hline A. angustifolia_200 & $*$ & & \\
\hline A. angustifolia_250 & $*$ & & \\
\hline A. angustifolia_300 & & $*$ & \\
\hline A. angustifolia_350 & & $*$ & \\
\hline A. angustifolia_400 & & $*$ & \\
\hline A. angustifolia_450 & & & $*$ \\
\hline A. angustifolia_500 & & & $*$ \\
\hline A. angustifolia_550 & & & $*$ \\
\hline A. angustifolia_600 & & & $*$ \\
\hline A. columnaris_200 & $*$ & & \\
\hline A. columnaris_250 & $*$ & & \\
\hline A. columnaris_300 & & $*$ & \\
\hline A. columnaris_350 & & $*$ & \\
\hline A. columnaris_400 & & $*$ & \\
\hline A. columnaris_450 & & & $*$ \\
\hline A. columnaris_500 & & & $*$ \\
\hline A. columnaris_550 & & & $*$ \\
\hline A. columnaris_600 & & & $*$ \\
\hline
\end{tabular}

Assim, a modelagem SIMCA criada a partir dos diferentes grupos separados pela PCA mostrou que pode vir a ser usada para prever a temperatura de queima aproximada de diferentes lenhos, independentemente de sua classe/espécie.

\section{CONCLUSÃO}

Os resultados obtidos nesse estudo evidenciaram que associações de técnicas como termogravimetria, espectroscopia de infravermelho médio e análise multivariada podem auxiliar na caracterização de processos de carbonização. Verificou-se que os lenhos carbonizados em mufla de cada uma das espécies analisadas apresentaram características térmicas semelhantes e carbonização na mesma faixa de temperatura. A termogravimetria permitiu identificação de eventos térmicos semelhantes durante o processo de termodegradação dos lenhos.

Ao avaliar os resultados da termodegradação dos lenhos para as três espécies estudadas, observou-se que o FTIR, associado à análise multivariada, constitui-se em ferramenta útil por permitir uma análise dos lenhos carbonizados, baseados na busca de semelhanças ou agrupamentos de amostras, a partir da similaridade entre as espécies avaliadas 
e suas respectivas faixas de temperaturas. A modelagem SIMCA criada a partir dos três grupamentos observados na PCA possibilitou a classificação de um conjunto externo de amostras com 100\% de acerto.

Com base nesses resultados, estão sendo realizados estudos objetivando a comparação com amostras de carvão vegetal macroscópico e carvão mineral para possíveis inferências paleoambientais.

\section{MATERIAL SUPLEMENTAR}

No material suplementar, disponível em http://quimicanova.sbq. org.br na forma de arquivo PDF, com acesso livre, encontra-se o detalhamento dos principais picos dos espectros presentes na Figura 3b (Tabela 1S). A representação dos scores para PC1 versus $\mathrm{PC} 2$ para as amostras de lenhos carbonizados artificialmente em mufla para a Araucaria angustifolia, Araucaria bidwillii e Araucaria columnaris estão apresentadas na Figura 1S.

\section{AGRADECIMENTOS}

Os autores agradecem aos técnicos Me. M. B. Horn e Dr. V. Ilha e à CAPES pelos auxílios financeiros concedidos, projetos CAPES (A072/2013), CNPq (301585/2012-1, 400972/2013-1, 444330/2014-3, 310421/2013-6). A. Jasper agradece à FAPERGS, ao CNPq (305436/2015-5; 444330/2014-3), à CAPES (Brasil - 810714-9) e à Alexander von Humboldt Foundation (Germany BRA 1137359 STPCAPES).

\section{REFERENCIAS}

1. Jasper, A.; Uhl, D.; Guerra-Sommer, M.; Mosbrugger, V.; J. South Am. Earth Sci. 2008, 26, 435.

2. Scott, A. C.; Stea, R.; Geoscientist 2002, 12, 1 .

3. Preston, C. M.; Schmidt, M. W. I.; Biogeosciences 2006, 3, 397.

4. Scott, A. C.; Palaeogeogr., Palaeoclimatol., Palaeoecol. 2010, $291,1$.

5. Scott, A.C.; Damblon, F.; Palaeogeogr., Palaeoclimatol., Palaeoecol. 2010, 291, 11 .

6. Scheel-Ybert, R.; Review of Palaeobotany and Palynology 2000, 110, 111 .

7. Bowman, D. M. J. S.; Balch, J. K.; Artaxo, P.; Bond, W. J.; Carlson, J. M.; Cochrane, M. A.; D'antonio, C. M.; Defries, R. S.; Doyle, J. C.; Harrison, S. P.; Johnston, F. H.; Keeley, J. E.; Krawchuk, M. A.; Kull, C. A.; Marston, J. B.; Moritz, M. A.; Prentice, I. C.; Roos, C. I.; Scott, A. C.; Swetnam, T. W.; Van Der Werf, G. R.; Pyne, S. J.; Science 2009, 324,481

8. Flannigan, M. D.; Krawchuk, M. A.; De Groot, W. J.; Wotton, B. M.; Gowman, L. M.; Int. J. Wildland Fire 2009, 18, 5.

9. Oliveira, M.; Manual de Estratégias, táticas e técnicas de combate a incêndios estruturais. Ed. Editograf: Florianopolis, 2005.

10. Scott, A. C.; Palaeogeogr., Palaeoclimatol., Palaeoecol. 2000, 164, 281

11. Jasper, A.; Guerra-Sommer, M.; Hamad, A. A. M. B.; Bamford, M.; Bernardes-De-Oliveira, M. E. C.; Tewari, R.; Uhl, D.; Gondwana Research 2013, 24, 148

12. Manfroi, J.; Uhl, D.; Guerra-Sommer, M.; Francischin, H.; Martinelli, A. G.; Soares, M. B.; Jasper, A.; Palaeogeogr., Palaeoclimatol., Palaeoecol. 2015, 436, 77.

13. Pappa, A.; Mikedi, K.; Tzamtzis, N.; Statheropoulos, M. J. Anal. Appl. Pyrolysis 2003, 67, 221.

14. Gómez, C. J.; Mészáros, E.; Jakab, E.; Velo, E.; Puigjaner, L.; J. Anal. Appl. Pyrolysis 2007 80, 416.

15. Risoluti, R.; Materazzi, S.; Sorrentino, F.; Maffei, L.; Caprari, P.; Talanta 2016, 159, 425.

16. Tomassetti, M.; Marini, F.; Bucci, R.; Coppa, A.; Campanella, L.; Microchem. J. 2016, 124, 396.
17. Moore, A. K.; Owen, N. L.; Appl. Spectrosc. Rev. 2001, 36, 1.

18. Chang, S. T.; Chang, H. T.; Polym. Degrad. Stab. 2001, 71, 2.

19. Colom, X.; Carrillo, F.; Nogues, F.; Garriga, P.; Polym. Degrad. Stab. 2003, 80,3 .

20. Schwanninger, M.; Rodrigues, J. C.; Pereira, H.; Hinterstoisser, B.; Vibrat. Spectrosc. 2004, 36, 1 .

21. Ascough, P. L.; Bird, M. I.; Scott, A. C.; Collinson, M. E.; Weiner, S.; Cohen-Ofri, I.; Snape, C. E. L.; Manquais, K.; J. Archaeol. Sci. 2010, 37,7 .

22. Minkina, M.; Zajusz-Zubek, E.; Mianowski, A.; Acta Geodyn. Geomater. 2006, 3, 2.

23. Batista, F. R. R.; Dissertação de Mestrado, Universidade Federal do Paraná, Curitiba, Brasil, 2016.

24. Labbeä, N.; Harper, D.; Rials, T.; J. Agric. Food Chem. 2006, 54, 3492.

25. Sebio-Puñal, T.; Naya, S.; López-Beceiro, J.; Tarrío-Saavedra, J.; Artiaga, R.; J. Therm. Anal. Calorim. 2012, 109, 1163.

26. Zodrow, E. L.; D’angelo, J. A.; Helleur, R.; Simunek, Z.; Int. J. Coal Geol. 2012, 100, 40.

27. O'keefe, J. M. K.; Bechtel, A.; Christanis, K. D.; Shifeng, D.; William, A.; Eble, C. F.; Esterle, J. S.; Mastalerz, M.; Raymond, A. L.; Valentim, B. V.; Wagner, N. J.; Colin, R.; Hower, J. C.; Int. J. Coal Geol. 2013, $118,58$.

28. D'angelo, J. A.; Escudero, L. B.; Volkheimer, W.; Zodrow, E. L.; Int. J. Coal Geol. 2011, 87, 2.

29. Helfer, G. A.; Bock, F.; Marder, L.; Furtado, J. C.; Costa, A. B.; Ferrão, M. F.; Quim. Nova, 2015, 38, 4 .

30. Allué, M. E.; Tese de Doutorado, Universidat Rovira i Virgili, Tarragona, Espanha, 2002.

31. Tereso, J. P. V.; Dissertação de Mestrado, Universidade do Porto, Porto, Portugal, 2007.

32. http://www.fao.org/docrep/X5555E/x5555e03.htm\#2.4 what happens during carbonization, acessado em Junho 2017.

33. Treusch, O.; Hofenauer, A.; Troger, F.; Fromm, J.; Wegener, G.; Wood Sci. Technol. 2004, 38, 323.

34. Rowell, R. M.; Pettersen, R.; Han, J. S.; Rowell, J. S.; Tshabalala, M. A. Em: Handbook of Wood Chemistry and Wood Composites; Rowell, R. M., ed.; CRC Press: Florida, 2005, cap. 3.

35. Bourgois, J.; Guyonnet, R.; Wood Sci. Technol. 1988, 22, 143.

36. Werner, K.; Pommer, L.; Broström, M.; J. Anal. Appl. Pyrolysis 2014, 110,130 .

37. Barneto, A. G.; Hernández, R. B.; Berenguer, J. M.; O Papel 2011, 72, 7.

38. Wiedman, H. G.; Riesen, R.; Boller, A.; Bayer, G. Em Thermogravimetry; Earnst, C. M., ed.; American Society for Testing and Materials: Philadelphia, 1988.

39. Nassarl, M. M.; MacKay, G. D. M.; Wood Fiber Sci. 1984, 16, 3.

40. Yang, H.; Yan, R.; Chen, H.; Lee, D.H.; Zheng, C.; Fuel 2007, 86, 1781.

41. Haykiri-Acma, H.; Yaman, S.; Kucukbayrak, S.; Fuel Process. Technol. 2010, $91,759$.

42. Rendeiro, G.; Nogueira, M. F. M.; Brasil, A. C. M.; Cruz, D. O. A.; Guerra, D. R. S.; Macêdo, E. M.; Ichiara, J. A.; Combustão e gasificação de biomassa sólida, Ministério de Minas e Energia, Brasília, 2008

43. Pinheiro, P. C. C.; Figueiredo, F. J.; Sèye, O.; Revista Biomassa \& Energia 2006, 2, 2.

44. Figueroa, M. J. M.; Moraes, P. D.; Ambiente Construído 2009, $9,4$.

45. Wiemann, M. C.; Williamson, G. B.; Wood Fiber Sci. 2002, 34, 1.

46. Baker, T. R.; Phillips, O. L.; Malhi, Y.; Almeida, S.; Arroyo, L.; Fiore, D. F.; Erwin, T.; Killeen, T. J.; Laurance, S. G.; Laurance, W. F.; Lewis, S. L.; Lloyd, J.; Monteagudo, A.; Neill, D. A.; Patiño, S.; Pitman, N. C. A.; Silva, J. N. M.; Martinez, R. V.; Global Change Biol. 2004, 10, 545.

47. Barbosa, L. C.; Espectroscopia no Infravermelho na Caracterização de Compostos Orgânicos. Ed. da UFV: Viçosa, 2007. 